\title{
Hydrocarbon injection for quantification of chemical erosion yields in tokamaks
}

\author{
S.Brezinsek $^{a *}$, A.Pospieszczyk ${ }^{a}$, D.Borodin ${ }^{a}$, M.F.Stamp $^{b}$, R.Pugno ${ }^{c}$, A.G.McLean ${ }^{d}$,

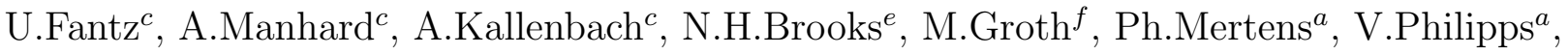 \\ U.Samm $^{a}$ and TEXTOR, ASDEX Upgrade, DIII-D teams and JET-EFDA contributors ${ }^{\star}$ \\ ${ }^{a}$ Institut für Plasmaphysik, Forschungszentrum Jülich, Association EURATOM-FZJ, \\ Trilateral Euregio Cluster, Germany, www.fz-juelich.de/ipp \\ ${ }^{b}$ Euratom/UKAEA Fusion Association, Culham Science Centre, Abingdon, Oxon OX14 3DB, UK \\ ${ }^{c}$ Max-Planck-Institut für Plasmaphysik, IPP-EURATOM Association, \\ "D-85748 Garching, Germany \\ ${ }^{d}$ University of Toronto, Department of Aerospace Studies, Canada \\ ${ }^{e}$ General Atomics, San-Diego, CA, USA \\ ${ }^{f}$ Lawrence Livermore National Laboratory, Livermore, USA \\ * See annex of J. Pamela et al., Fusion Energy 2002 (19 ${ }^{\text {th }}$ Int. Conf., Lyon, 2002), IAEA, Vienna
}

\begin{abstract}
Chemical erosion of carbon can be described as function of the incident ion flux, the ion energy and the surface temperature. Spectroscopy on the hydrocarbon break-up products $\mathrm{CH}$ and $\mathrm{C}_{2}$ is applied to determine in-situ hydrocarbon fluxes and quantify the chemical erosion by means of hydrocarbon injection. Present-day knowledge on critical issues concerning the erosion yield and its determination is presented: Effective inverse photon efficiencies are measured in TEXTOR for different hydrocarbon species and compared with calculations from HYDKIN. The underlying database for the methane break-up, used in different erosion/deposition models, has been put to test. The chemical erosion yield related to higher hydrocarbons is determined to be lower in the JET outer divertor than measured in previous experiments. In ASDEX Upgrade and DIII-D first in-situ calibrations of hydrocarbon fluxes in the detached outer divertor are performed.
\end{abstract}


PACS: 52.70.Kz, 52.40.Hf, 52.25.Vy, 52.55.Fa, 33.20.Lg

JNM keywords: Carbon, Impurities, Plasma-Materials Interaction, Redeposition, Surface effects

PSI-16 keywords: Spectroscopy, ITER, Carbon impurities, Erosion \& Deposition, Divertor * Corresponding author address: Institut für Plasmaphysik, Forschungszentrum Jülich GmbH, D-52425 Jülich, Germany

*Corresponding author e-mail: s.brezinsek@fz-juelich.de

Presenting author: Sebastijan Brezinsek

Presenting author e-mail: s.brezinsek@fz-juelich.de 


\section{Introduction}

The use of carbon-based materials for plasma-facing components (pfc) in fusion devices is connected to the appearance of chemical erosion, followed by carbon migration and deposition. The main chamber has been identified as one of the main sources of eroded carbon, and marker experiments indicated a stepwise transport of carbon towards the depositiondominated inner divertor [1]. The outer divertor often exhibits balanced erosion/deposition, though net erosion has been observed in some devices [2]. The experimental determination of carbon fluxes -atomic and molecular ones- is crucial for the understanding of migration and verification of erosion/deposition codes [3] which are used for predictions of erosion and tritium co-deposition in the ITER divertor.

Passive emission spectroscopy has access to fluxes $\Gamma_{C}$ of carbon in different ionisation states as well as of molecular fragments of hydrocarbons, in particular $C H$ and $C_{2}$ radicals, and thus, to the physical sputtering $Y_{C}^{\text {phys }}$ and chemical erosion yield $Y_{C}^{c h e m}$. $Y_{C}^{\text {chem }}$ can be described as a function of ion energy, ion flux and surface temperature [4], though uncertainties with respect, among others, to data interpretation, higher hydrocarbons and extrapolation to detached plasma regimes exist.

Essential for the $Y_{C}^{c h e m}$ is the quantification of $\Gamma_{C}^{c h e m}$ which is made with the aid of photon fluxes $\phi$ of hydrocarbon break-up products and effective inverse photon efficiencies. This indirect conversion includes the dissociation chain and has been identified as a critical issue. In-situ calibration with injection of hydrocarbons takes into account local conditions like plasma parameters, surface properties and geometry.

\section{The spectroscopic approach to determine $\Gamma_{C}^{\text {chem }}$ and $Y_{C}^{\text {chem }}$}

Spectroscopy is used to determine particle fluxes in fusion devices [5]. Photon fluxes are converted into particle fluxes with the aid of inverse photon efficiencies, or so-called S/XB or $\mathrm{D} / \mathrm{XB}$ values. There $\mathrm{S}$ stands for the ionisation rate coefficient of an atom, $\mathrm{D}$ for the 
decay rate coefficient of a molecule which represents its "loss" by either dissociation or ionisation, and $\mathrm{XB}$ is the excitation rate coefficient of the observed transition weighted with the branching ratio for a given set of electron density $n_{e}$ and temperature $T_{e}$.

The carbon flux $\Gamma_{C}^{\text {total }}=\Gamma_{C}^{c h e m}+\Gamma_{C}^{\text {phys }}$ can be deduced by observation of carbon ion radiation, i.e. CII, and due consideration of corresponding $\mathrm{S} / \mathrm{XB}$ values from ADAS [6]. Although this emission is localised in the plasma edge, no information about the source processes, whether involving physical or chemical sputtering, can be obtained. The hydrocarbon flux $\Gamma_{C}^{c h e m}$ and with it $Y_{C}^{\text {chem }} \simeq \frac{\Gamma_{C}^{\text {chem }}}{\Gamma_{D}}$, the flux ratio of chemically eroded carbon to the impinging hydrogen, is needed to distinguish between these processes. The recycling flux $\Gamma_{D}[7]$ is assumed to be equivalent to the impinging ion flux.

\subsection{Photon fluxes and effective inverse photon efficiencies for hydrocarbons}

The carbon flux $\Gamma_{C}^{c h e m}$ originates from molecules of the methane $\left(C H_{x}\right.$ with $\left.x \leq 4\right)$, the ethane $\left(C_{2} H_{y}\right.$ with $\left.y \leq 6\right)$ and the propane family $\left(C_{3} H_{z}\right.$ with $\left.z \leq 8\right)$ and is given by $\Gamma_{C}^{c h e m}=\Gamma_{C}^{C H_{x}}+2 \Gamma_{C}^{C_{2} H_{y}}+3 \Gamma_{C}^{C_{3} H_{z}}+\ldots$. The initial hydrocarbon as well as the main part of molecular ions or radicals built up along the complex destruction path [8] in the plasma cannot be detected by emission spectroscopy. Only $\mathrm{CH}, \mathrm{CH}^{+}$and $\mathrm{C}_{2}$ can be observed; the $\mathrm{CH}$ Gerö band, main representative for $\mathrm{CH}_{x}$, the $C_{2}$ Swan band, representative for $C_{2} H_{y}$, and the $\mathrm{CH}^{+}$Douglas-Herzberg band are the main recorded transitions in fusion-related plasmas [9]. Effective D/XB values include the dissociation chain for given plasma parameters [10]. For $\mathrm{CH}_{x}$ and $\mathrm{C}_{2} \mathrm{H}_{y}$ the important ones are:

$$
\begin{aligned}
& {\left[\frac{D}{X B}\right]_{\mathrm{A}^{2} \Delta \rightarrow \mathrm{X}^{2} \Pi}^{C H_{x} \rightarrow C H}=\frac{\Gamma^{C H_{x}}}{\phi_{\mathrm{A}^{2} \Delta \rightarrow \mathrm{X}^{2} \Pi}^{C H_{x} \rightarrow C H}}} \\
& {\left[\frac{D}{X B}\right]_{\mathrm{d}^{3} \Pi \rightarrow \mathrm{a}{ }^{3} \Pi}^{C_{2} H_{y} \rightarrow C_{2}}=\frac{\Gamma^{C_{2} H_{y}}}{\phi_{\mathrm{d}^{3} \Pi \rightarrow \mathrm{a}^{3} \Pi}^{C_{2} H_{y} \rightarrow C_{2}}} \text { and }\left[\frac{D}{X B}\right]_{\mathrm{A}^{2}{ }^{2} \rightarrow \mathrm{X}{ }^{2} \Pi}^{C_{2} H_{y} \rightarrow C H}=\frac{\Gamma^{C_{2} H_{y}}}{\phi_{\mathrm{A}{ }^{2} \Delta \rightarrow \mathrm{X}{ }^{2} \Pi}^{C_{2} H_{y} \rightarrow C H}}}
\end{aligned}
$$

Fig.1a shows examples of spectra and simulations for the Douglas-Herzberg and the Gerö bands. Reduced spectral ranges are chosen to cover a contamination-free representative portion of the band. Different wavelength ranges $\left(\lambda_{\text {span }}\right)$ for the $C D$ Gerö band are in use: 
429.5-431.0nm at TEXTOR [10], ASDEX Upgrade [11] and JT-60U [12], which cover the first $1.5 \mathrm{~nm}$ at the band head, as well as 427.0-431.5nm at DIII-D [13] and 427.8-431.5nm at JET [14].Data for the $C H$ and $C D$ Gerö band presented here are normalised to the full spectral range of the electronic transition (spanning: 415.0-445.0nm) by multiplying with calculated expansion factors $f_{A-X}^{C H}$ or $C D\left(T_{\text {rot }}, \lambda_{\text {span }}\right)[10,11]$ except where other indications are made. These expansion factors are determined by the ratio of the light emission in $\lambda_{\text {span }}$ to the total light emission of the A-X band which can be obtained from spectra fitting or simulation for a given ro-vibrational population [15]. Data for the $C_{2}$ Swan band are normalised in a similar way with the aid of $f_{d-a}^{C_{2}}\left(T_{\text {rot }}, \lambda_{\text {span }}\right)$ (see sec.3).

\subsection{Experimental realisation of injection experiments}

Calibration experiments with injection of stable hydrocarbons are carried out to determine effective $\mathrm{D} / \mathrm{XB}$ values and, thus, to relate the emitted photon flux of hydrocarbon fragments to the flux of injected hydrocarbon particles. The individual injection rate is chosen high enough to ensure significant photon fluxes from the injection with respect to the intrinsic background and low enough to minimise plasma disturbances. Apart from JET, where a multiple injection through toroidally circumferential gas inlets is made [14], all other experiments presented here are done with single injections.

In the case of circumferential injection at JET $\left(\sim 2 \cdot 10^{21} \frac{\mathrm{part} .}{\mathrm{s}}\right)$, a toroidally homogeneous distribution of the injected gas in the observation volume is assumed. The edge plasma is disturbed and reference discharges are necessary to take changes in the hydrocarbon source strength into account. Experimentally determined quantities are averages over large areas. Note that recent experiments in DIII-D with circumferential injection of methane through the upper plenum were performed without significant disturbance of the edge plasma parameters [16].

In the case of single gas injections $\left(0.5-15 \cdot 10^{18 \frac{\mathrm{part} .}{\mathrm{s}}}\right)$, a perturbation of the local plasma 
parameters at a representative location occurs but reference discharges are unnecessary due to the low degree of perturbation. The observation volume has to be chosen large enough to ensure that all photons induced by the gas injection are detected. Transfer to other locations might be affected by uncertainties [17]. At ASDEX Upgrade [18] and DIII-D [19] the gas injection systems, discussed here, are integral part of the outer divertor and thus embedded in carbon-based target plates. The porous-plug injector PPI is in particular in use to simulate the chemically eroded carbon by injection through micro holes. At TEXTOR, different injection systems, either integrated in limiters or in metallic tubes, have been applied in a flexible vacuum lock system [7].

\section{Experimentally determined and calculated effective inverse photon efficiencies}

Spectrum simulations of the Gerö and the Swan band in different experiments have shown that the ro-vibrational populations, and therewith the expansion factors, are quite robust and vary only slightly with plasma parameters. Typical rotational temperatures have been found for a wide range of plasma parameters: 3500+/-500 K for the Gerö band with $f_{A-X}^{C D}\left(T_{\text {rot }}=3500 K, \lambda_{\text {span }}=429.5 \mathrm{~nm}-431.0 \mathrm{~nm}\right)=2.8$ and $f_{A-X}^{C H}\left(T_{\text {rot }}=3500 \mathrm{~K}, \lambda_{\text {span }}=\right.$ $430.0 \mathrm{~nm}-431.5 \mathrm{~nm})=2.8$ for the complete $A-X$ transition and $3000+/-1000 \mathrm{~K}$ for the Swan band with $f_{d-a}^{C_{2}}\left(T_{\text {rot }}=3000 K, \lambda_{\text {span }}=515.3 \mathrm{~nm}-516.6 \mathrm{~nm}\right)=4$ for the main diagonals $(\Delta v=0$ with $v=0-3)$ of the $d-a$ transition. The origin of the $C_{2}$ molecule seems to plays no role, even sublimated $C_{2}$, observed at an accidently overheated graphite protection tile in TEXTOR, shows for comparable plasma conditions an almost identical ro-vibrational population in comparison to $C_{2}$ from hydrocarbon injections (fig.1b).

\subsection{Effective D/XB values for different hydrocarbons in TEXTOR}

A benchmark experiment in TEXTOR has been performed to validate the underlying data base for the hydrocarbon break-up [8] used in the ERO code. Different hydrocarbons were injected in $D$ or $H$ plasmas. The use of different isotopes allows to distinguish between $C D$ 
and $C H$ as well as between $D$ and $H$ from the break-up and the plasma background. The observation volume was chosen large enough to ensure that molecular hydrocarbon fragments of type $\mathrm{CH}$ or $C_{2}$ are either dissociated or ionised before leaving the observation volume. Losses due to transport out of the volume can be excluded. 2D images from top and from the side recorded with intensified CCD cameras equipped with interference filters for the observed transitions were used for verification (fig.2a). Gas inlet and observation volume were free of graphite surfaces, thus no intrinsic neutral and molecular carbon was detectable prior to the injection. Moreover, no significant surface area for local deposition exists within the observation volume under consideration of the short penetration depth of the fragments (fig.2b). The observed light emission can be referred completely to the bare impact of the plasma on the injected hydrocarbons; secondary effects caused by either sinks or sources of hydrocarbons at surfaces can be excluded.

$\mathrm{D} / \mathrm{XB}$ values for the different injected species (tab.1) were related to the edge plasma parameters at the position of maximum light emission (fig.2b). These are in the case of $D$ plasmas: $n_{e}=2.2 \cdot 10^{18} \mathrm{~m}^{-3}$ and $T_{e}=35 \mathrm{eV}$ and in the case of $H$ plasmas: $n_{e}=1.8 \cdot 10^{18} \mathrm{~m}^{-3}$ and $T_{e}=45 \mathrm{eV}$ as taken from the outer midplane and measured by Langmuir probes [20] or He-beam diagnostics [21]. These non-local edge plasma parameters were not influenced by the hydrocarbon injection. Local measurement of $T_{e}$ by a Langmuir probe, installed for comparable experiments directly next to the gas inlet, indicate a slight reduction of $T_{e}$ of less than $15 \%$ during the injection. This grade of disturbance is in-line with $T_{e}$ measurements during the hydrocarbon injection obtained from the analysis of the Balmer- $\beta$ to Balmer- $\gamma$ line ratio in the emission cloud of the background plasma. The gas inlet was positioned $1.5 \mathrm{~cm}(\mathrm{H})$ and $2.0 \mathrm{~cm}(\mathrm{D})$ behind the LCFS. The uncertainty in each effective $\mathrm{D} / \mathrm{XB}$ values is below $20 \%$.

D/XB values for $C H$ and $C D$ denoted with $J R$ are calculated with the aid of HYDKIN [22], 
a reaction kinetic analysis solver for the catabolism of hydrocarbon in hydrogen plasmas, and vibrationally resolved emission rate coefficients for the Gerö band. These vibrationally resolved emission rate coefficients are 1.48 times larger than the non-vibrational resolved ones [11] which are ordinary applied in HYDKIN (memo in [22]). The effective D/XB values presented here are diminished by this factor 1.48 in comparison with the standard HYDKIN output.

HYDKIN assumes a constant plasma background as input parameter. The calculation of $\mathrm{D} / \mathrm{XB}$ values is reasonable as long as the variation of the involved rate coefficients is small in the plasma parameter range where the molecular fragments exist. The ERO code [3] takes additionally the transport, the edge plasma parameters profiles and the local geometry into account. Modelling has so far only been applied for $C D_{4}$ (fig.2c). $\left[\frac{D}{X B}\right]_{\mathrm{A}}^{C D^{2} \Delta \rightarrow C D}{ }^{2} \Pi$ from ERO amounts to 32 and is in agreement with the experimental value of 36, and slightly lower than the HYDKIN value of 46 . Thus, the database for the methane break-up [8] is reliable in the plasma parameter range of TEXTOR. However, ERO modelling is needed for an exact interpretation of the injection experiments, but HYDKIN calculations can be applied as a step in testing the database.

Photon efficiencies for $\mathrm{C}_{2}$ and $\mathrm{CH}$ from $\mathrm{C}_{2} H_{y}$ and $\mathrm{C}_{3} H_{z}$ calculated with HYDKIN and the emission rate coefficient for the Swan band [11] are comparable with the measured values, they differ at most by a factor of 2 , where less $C H$ light and more $C_{2}$ light is predicted from calculations. This indicates uncertainties in the database for $C_{2} H_{y}$ and $C_{3} H_{z}$ with respect to the branching ratio of higher hydrocarbons.

Information about the break-up reactions can be deduced from effective inverse photon efficiencies for $C, C^{+}$, and $H$, build along the dissociation chain. These conversion factors, normalised to the number of $C$ atoms or $H$ atoms in the initial hydrocarbon, can be related to bare $\mathrm{S} / \mathrm{XB}$ values from ADAS for atoms and ions. The ratio $\left[\frac{S}{X B}\right]_{A D A S} /\left[\frac{D}{X B}\right]_{E X P}$ for 
the different fragments and their electronic transitions is defined as the normalised photonper-particle production efficiency $\eta$. For $\mathrm{CH}_{4}$ the following values $\left[\frac{D}{\mathrm{XB}}\right]_{\mathrm{CI} \text { 909.5nm }}^{C H_{4} \rightarrow C}=72$ with $\eta=0.20,\left[\frac{D}{X B}\right]_{\mathrm{CII} 426.7 \mathrm{~nm}}^{C H_{4} \rightarrow C^{+}}=277$ with $\eta=0.56,\left[\frac{D}{X B}\right]_{\mathrm{H}_{\gamma}}^{C H_{4} \rightarrow H}=390$ with $\eta=0.19$ were determined.

$\eta<1$ means that less photons per particle are observed during the break-up than expected from the bare number of $C$ or $H$ particles in the initial hydrocarbon. For example, the number of $C I(909.5 \mathrm{~nm})$ photons emitted per $\mathrm{CH}_{4}$ launched into the plasma is only $20 \%$ of the number that would be calculated using ADAS per isolated $C$ atom launched into the same plasma. Either less photons are emitted, e.g. due to dissociation of a molecular fragment into an atom or ion which is directly excited in a higher electronic state than the upper state of the observed transition, or more likely, less fragments of the observed kind are produced during the catabolism, e.g. absence of neutral $C$ due to dissociation of $C D^{+}$into $D$ and $C^{+}$. In the case of hydrogen: the absence of a large fraction of photons per atom $(\eta \simeq 0.2)$ has been observed transition-independently for $H_{\beta}, H_{\gamma}$, and $H_{\delta}$. Only about $20 \%$ of the expected hydrogen atoms have been detected, thus probably more protons than atoms are built up during the break-up. In the case of the carbon balance: on the one hand only $20 \%$ of the theoretically possible $C I(909.5 \mathrm{~nm})$ photons per neutral $C$ are detected, and on the other hand the number of $C I I(426.7 \mathrm{~nm})$ photons per $C^{+}$is about a half that theoretically possible. However, more $C I$ and $C I I$ transitions have to be analysed to discriminate the two possible effects mentioned above, the large difference in the efficiency for $C$ and $C^{+}$ indicates that the destruction path, though it is a multi-step process, goes substantially via molecular ions, which is in-line with HYDKIN calculations and the prominent presence of the $C D^{+} \mathrm{A}-\mathrm{X}$ band (fig.1a) in TEXTOR.

\subsection{Effective $\mathrm{D} / \mathrm{XB}$ values for methane from different devices}

A set of data from different experiments exists for ohmic or L-mode discharges with injec- 
tion of $\mathrm{CH}_{4} / \mathrm{CD}_{4}$ into outer divertor plasmas. At JET the injection was into the private

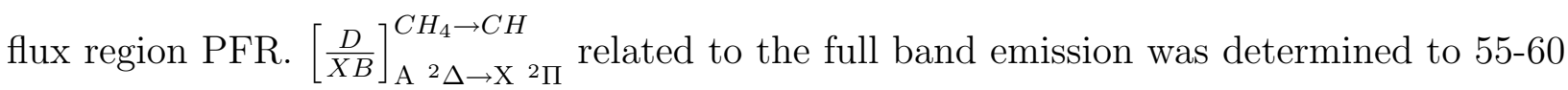
$[10,23]$ when the outer-strike point was on the vertical target $\left(T_{e}=40 \mathrm{eV}, n_{e}=6 \cdot 10^{19} \mathrm{~m}^{-3}\right)$. Though the toroidally homogeneity of the injection cloud is given in vertical configuration, a minor loss of particles into the inner divertor and to the pump duct of the outer divertor cannot be excluded and $\left[\frac{D}{X B}\right]_{\mathrm{A}^{2} \Delta \rightarrow \mathrm{X}{ }^{2} \Pi}^{C H_{4} \rightarrow C H}=55$ is an upper limit. Similar values, but for lower $T_{e}$ were reported in previous experiments [14].

At DIII-D the injection was into the scrape-off layer (SOL) $\left(T_{e}=22.5 \mathrm{eV}, n_{e}=2.5 \cdot 10^{19} \mathrm{~m}^{-3}\right)$ and $\left[\frac{D}{X B}\right]_{\mathrm{A}^{2}{ }^{2} \Delta \rightarrow \mathrm{X}{ }^{2} \Pi}^{C H_{4} \rightarrow C H}$ was determined to $40+/-11[19]$ when normalised to the full band. At ASDEX Upgrade methane injection into the PFR and SOL for high density hydrogen plasmas is well described [24]. The D/XB value related to the Gerö band varies between 4 and 20 for $n_{e}=2 \ldots 8 \cdot 10^{19} \mathrm{~m}^{-3}$ and $\left.T_{e}=5 \ldots 15 \mathrm{eV}\right)$.

Fig.3 shows an overview of $\left[\frac{D}{X B}\right]_{\mathrm{A}^{2}{ }^{2} \rightarrow \rightarrow \mathrm{X}{ }^{2} \Pi}^{C H_{4} \rightarrow C H}$ and $\left[\frac{D}{X B}\right]_{\mathrm{A}}^{C D_{4} \rightarrow C D}{ }^{2} \Delta \rightarrow \mathrm{X}{ }^{2} \Pi$ from the different experiments compared with calculated values (HYDKIN) as function of $T_{e}$. Additional experimental data, in particular from TEXTOR, was added and, for clarification, in some cases only representative points were taken from a larger set of data. The corresponding $\lambda_{\text {span }}$ and $f\left(T_{\text {rot }}, \lambda_{\text {span }}\right)$ values which were applied for the normalisation are given in tab.2.

Though different local conditions and geometries are involved, the general increase of $\left[\frac{D}{X B}\right]_{\mathrm{A}^{2} \Delta \rightarrow \mathrm{X}^{2} \Pi}^{C H_{4} \rightarrow C H}$ with $T_{e}$, as calculated with HYDKIN, is well reproduced, indicating both the reliability of the underlying database for the methane break-up chain and the general consistency of experimental results over a wide plasma parameter range. However, in detail the experimental data with graphite surrounding shows higher effective $\mathrm{D} / \mathrm{XB}$ values, which means less light is observed than expected from HYDKIN. This discrepancy might be attributed to the simplified assumption of a constant plasma, to a loss of particles during the injection due to deposition or transport, or to changes in local conditions. For a more 
detailed comparison ERO calculations for each experiment are needed which are here out of the scope.

\subsection{Injection-induced re-erosion of higher hydrocarbons}

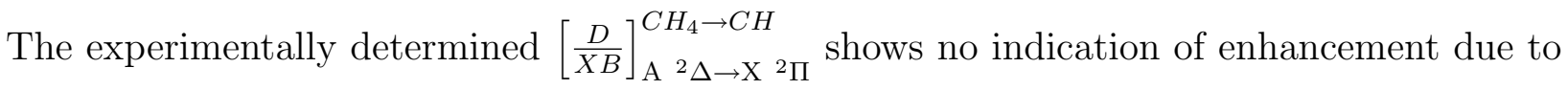
an additional source in the presence of a graphite surrounding. However, experiments with $\mathrm{CH}_{4}$ injection show an accompanying emission of $\mathrm{C}_{2}$ light when the injection was through gas inlets surrounded by graphite or when graphite layers are present.

In fig.4 the time evolution of $\phi_{d-a}^{C_{2}}$ during methane injection $\left(3 \cdot 10^{18} \frac{\text { part. }}{s}\right)$ in the attached outer divertor of ASDEX Upgrade is shown. The outer strike point was swept twice over the local injection. Low intrinsic $\phi_{d-a}^{C_{2}}$ was detected in the reference phase, whereas with $C H_{4}$ injection a strong and nearly instantaneous increase of $\phi_{d-a}^{C_{2}}$ up to a factor six was observed. The present understanding is: a fraction of the injected methane is locally deposited and immediately, with a high erosion rate re-eroded, substantially as higher hydrocarbon. In the consecutive discharge, apart from an increased emission during the strike-point formation no enhanced emission of $C_{2}$ light was observed. These observations are similar to experiments with the PPI [19], where also an increase of $\phi_{d-a}^{C_{2}}$ with methane injection was measured. The $C_{2}$ light decreased in the subsequent discharges without injection. This dynamic process as well as the contribution of possible re-eroded methane to the $C H$ light emission is a topic of present modelling with erosion deposition codes.

\section{Hydrocarbon fluxes and erosion yields}

Because of the limited space I confine myself on three examples, one which deals with the contribution of ethene to $Y_{C}^{c h e m}$, one which indicates the improved understanding of data analysis, and one which investigates the hydrocarbon flux in detached divertor plasmas.

\subsection{The contribution of $C_{2} H_{y}$ to $Y_{C}^{c h e m}$ in the JET outer divertor}

In JET, calibration experiments were made in deuterium discharges (L-mode, $T_{e}=25 \mathrm{eV}$ 
and $n_{e}=5.5 \cdot 10^{19} \mathrm{~m}^{-3}$ ) with circumferential puffing of ethene through an injection module (GIM10) located between the vertical plates of the outer divertor. The outer strike point was swept over the injection locations, thus $C_{2} H_{4}$ was either injected in the near, the far SOL or in PFR [25]. A reference discharges with hydrogen injection was performed to match the plasma parameters. The injection through GIM10 into the scrape-off layer suffer from a gas bypass $(\sim 25 \%$ loss $)$, the non-homogeneity of the injection $(\leq 40 \%$ of the expected gas reaches the observation volume) as well as from the cross-divertor transport $(\sim 10 \%$ loss $)$ when the outer strike point is positioned near to the injection location. This has been recently identified in tracer injection experiments with the outer-strike point portioned slightly below GIM10 by the analysis of the local deposited ${ }^{13} C[26]$. Analysis and simulations are ongoing to determine the exact portion of injected gas reaching the observation volume described in [14].

However, an upper limit for the effective inverse photon efficiency $\left[\frac{D}{X B}\right]_{\mathrm{d}}^{C_{2}{ }^{3} H_{4} \rightarrow C_{2}{ }^{3} \Pi}$ for the $C_{2}$ Swan band $\left(f_{d-a}^{C_{2}}\left(T_{\text {rot }}=3000 K, \lambda_{\text {span }}=515.3 n m-516.6 n m\right)=4\right)$ is determined to be below 75. Fig.5 shows the erosion yield distribution with the strike-point fixed on the poloidal location of GIM10. The erosion yield $Y_{C}^{\text {chem, } C_{2} H_{y}}$ is below $0.6 \%$ at the location with maximum impinging ion flux of about $1.65 \cdot 10^{23} \frac{\text { ions }}{s}$ at a surface temperature of about $450 \mathrm{~K}$. This is essentially less than reported in [14] where the injection was in the PFR. However, it is still above the predictions for the erosion yield from [3-4] which is for these conditions about $0.3 \%$.

\subsection{The total sputtering yield in TEXTOR}

In TEXTOR a pre-heatable spherical graphite limiter was used to determine the erosion yield as function of the graphite temperature. The limiter was positioned at the LCFS $\left(n_{e}=5.2 \cdot 10^{18} \mathrm{~m}^{-3}, T_{e}=42 \mathrm{eV}, T_{i}=150 \mathrm{eV}, \Gamma_{D^{+}}=7.5 \cdot 10^{22}\right.$ ions $\left.\mathrm{s}^{-1} \mathrm{~m}^{-2}\right)$ and pre-heated to $520 \mathrm{~K}$ before exposed to the plasma. Details on the applied spectroscopic systems and the 
experimental conditions can be found in [7].

Fig. 6a shows the behaviour of $C D$ and $D_{\gamma}$ intensity, with variation of the bulk temperature under otherwise constant plasma parameters. Clearly, the decrease of $C D$ intensity with higher surface temperatures and its complete absence at $1300 \mathrm{~K}$ can be observed [27]. Also indicated is the behaviour of deuterium molecules, which disturbed the $A-X$ band and which were taken into account in the analysis [7]. However, above $1100 \mathrm{~K}$ an increase of the $D_{\gamma}$ intensity can also be observed. Deuterium starts to be directly released as atoms and not as molecules from the surface. The deuterium recycling flux can be obtained from the extrapolated $D_{\gamma}$ maximum value, where the release is pure atomic [7]. The intensity ratio of $D_{\gamma}$ from the maximum value to $C D$, multiplied with the ratio of the $\mathrm{S} / \mathrm{XB}$ value for $D_{\gamma}$

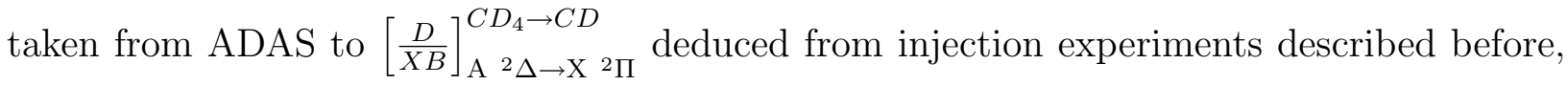
provides $Y_{C}^{c h e m}$. Due to this correction the erosion yield is reduced from about $Y_{C}^{c h e m}=4.0 \%$ to $Y_{C}^{\text {chem }}=3.0 \%$ in comparison to $[27]$.

Fig.6b shows $Y_{C}^{\text {total }}$ deduced from the light intensity of $C I I$ at $426.7 \mathrm{~nm}$. The difference at highest temperatures, where $Y_{C}^{\text {chem }}$ vanishes, represents $Y_{C}^{\text {phys }}$. In the case of TEXTOR $Y_{C}^{\text {chem }}$ and $Y_{C}^{\text {phys }}$ are almost balanced at the temperature of maximum erosion.

Although the yield determination is based only on the $C D$ photon flux, contributions from other hydrocarbons than $C D_{4}$ are implicitly included: $\phi_{\mathrm{A}}^{C{ }^{2} \Delta \rightarrow \mathrm{X}{ }^{2} \Pi}=\phi_{\mathrm{A}}^{C D^{2} \Delta \rightarrow C D \mathrm{X}^{2} \Pi}+$ $\phi_{\mathrm{A}{ }^{2} \Delta \rightarrow \mathrm{X}^{2} \Pi}^{C_{2} D_{y} \rightarrow C D}+\ldots$ Conversion using $\left[\frac{D}{X B}\right]_{\mathrm{A}^{2} \Delta \rightarrow \mathrm{X}{ }^{2} \Pi}^{C D_{x} \rightarrow C D}$ leads to the apparent particle flux $\tilde{\Gamma}_{C}^{C D_{x}}$ which overestimates $\Gamma_{C}^{C D_{x}}$ by the contribution of $\Gamma_{C}^{C_{2} D_{y}}$ to the $C D$ photon flux.

$\Gamma_{C}^{c h e m}$ can be described as function of the measured $C D$ and $C_{2}$ photon fluxes:

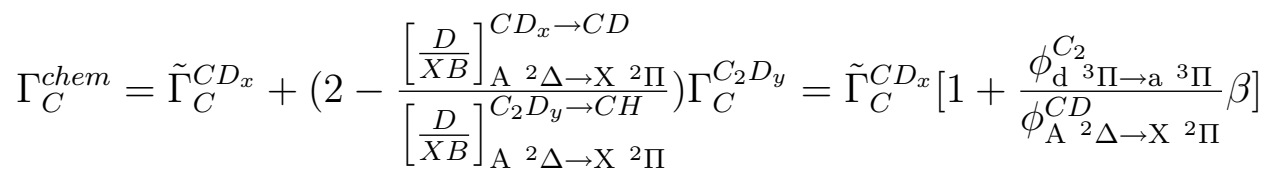

The correction of the apparent particle flux depends on $\phi_{\mathrm{d}^{3} \Pi \rightarrow \mathrm{a}{ }^{3} \Pi}^{C^{2}} / \phi_{\mathrm{A}}^{C D}{ }^{2} \Delta \rightarrow \mathrm{X}{ }^{2} \Pi$ and on the

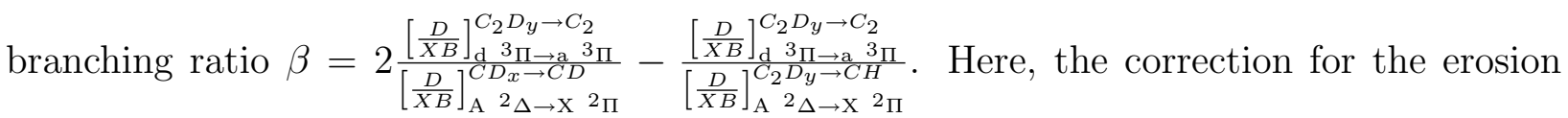
yield deduced from $C D$ emission is about 1.1, whereas $\phi_{\mathrm{d}^{3} \Pi \rightarrow \mathrm{a}{ }^{3} \Pi}^{C_{2}} \simeq 0.1 \phi_{\mathrm{A}}^{C D}{ }^{2} \Delta \rightarrow \mathrm{X}{ }^{2} \Pi$ and 
$\beta \simeq 1$. Thus, the measured $C D$ light underestimates the total erosion yield by about $10 \%$.

Note that we have omitted in this approach an essential production of carbon dimers out of two methane break-up products as well as contributions from $C_{3} D_{z}$. In the case of the attached ASDEX Upgrade divertor in L-mode $\left(T_{e} \simeq 5-20 \mathrm{eV}\right)$, the correction term is of the order of 1.3 , whereas $\beta$ is about 1 and $\phi_{\mathrm{d}^{3} \Pi \rightarrow \mathrm{a}{ }^{3} \Pi}^{C{ }} / \phi_{\mathrm{A}}^{C{ }^{2} \Delta \rightarrow \mathrm{x}{ }^{2} \Pi} \simeq 0.3[11]$.

\subsection{Hydrocarbon flux in detached plasmas}

The operational regime for the outer divertor in ITER is a detached plasma. Not much information about the chemical erosion, the hydrocarbon fluxes and the effective inverse photon efficiencies for this regime is available yet. Previous experiments in DIII-D [28] have shown a reduction of the light emission of the $C_{2}$ Swan band and of the $C H$ Gerö band when the outer divertor detaches under L-mode conditions. The reduction was attributed to the reduction of $\Gamma_{C}^{\text {chem }}$ and $Y_{C}^{\text {chem }}$, though the effective $D / X B$ values for $C H_{x}$ and $C_{2} H_{y}$ in detached plasma conditions were uncertain. Previous modelling predictions [28 and references therein] indicated for methane $\left[\frac{D}{X B}\right]_{\mathrm{A}^{2}{ }^{2} \Delta \rightarrow \mathrm{X}{ }^{2} \Pi}^{C H_{4} \rightarrow C H}=5$, which is comparable with HYDKIN calculations for $T \simeq 1 e V, n_{e} \simeq 2 \cdot 10^{20} m^{-3}$.

In ASDEX Upgrade, experiments in L-mode with detachment of the outer divertor by means of strong deuterium puffing were performed. Two sweeps of the outer strike-point over the gas injection location were made. Whilst the first one is used as reference, the second one is for the in-situ calibration of the photon fluxes with by $\mathrm{CH}_{4}$ and $\mathrm{C}_{2} \mathrm{H}_{4}$ injection. In detachment a strong reduction of both the intrinsic photon flux of $\phi_{A-X}^{C D}$ (fig.7) and $\phi_{d-a}^{C_{2}}$ of more than a factor 12 was observed in comparison to an attached plasma reference. In the case of the $C D$ A-X transition in detachment, strong disturbance (up to $50 \%$ ) by the $B D \mathrm{~A}-\mathrm{X}$ transition was found and taken into consideration in the analysis.

With injection of either $\mathrm{CH}_{4}$ or $\mathrm{C}_{2} \mathrm{H}_{4}$, a clear increase in the light emission was observed.

The corresponding effective inverse photon efficiencies were determined to $\left[\frac{D}{X B}\right]_{\mathrm{A}}^{C H_{4} \rightarrow C H}{ }^{2} \Delta \rightarrow \mathrm{X}{ }^{2} \Pi=$ 
$18+/-7$ and $\left[\frac{D}{X B}\right]_{\mathrm{A}}^{C_{2}{ }^{2} \Delta \rightarrow \mathrm{X}^{2} \Pi}=47+/-19$. The value for methane is about 1.2-4.0 times higher than reported in [24] for high density L-mode discharges with attached outer divertor. An increase of the emission zone of the injected species was detected and the loss of photons was estimated. However, the $\mathrm{D} / \mathrm{XB}$ values represent upper limits and further modelling is required.

Qualitatively similar results with stronger reduction of intrinsic light in divertor detachment were obtained in DIII-D under comparable plasma conditions [19]. Methane injection with

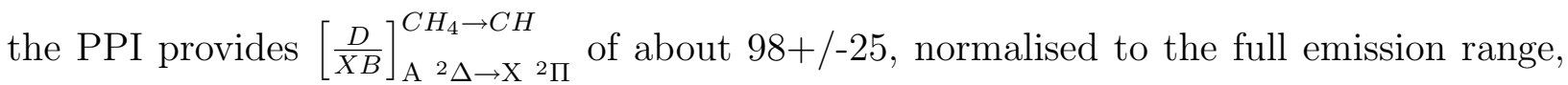
and thus about 2.5 larger in comparison to a typical attached case. Both experiments indicate that indeed the reduction of $\phi_{A-X}^{C D}$ (fig.7) and $\phi_{d-a}^{C_{2}}$ is accompanied by a reduction of the eroded hydrocarbon particle flux when the plasma detaches. The question, whether the flux reduction is linked to a reduction of $Y_{C}^{c h e m}$, is topic of a forthcoming paper.

\section{Summary and conclusion}

Passive emission spectroscopy was applied to determine effective inverse photon efficiencies, hydrocarbon fluxes and erosion yields in different fusion devices. This was achieved by means of in-situ calibration with hydrocarbon injection - either with a single inlet or with systems distributed on the circumference. Effective inverse photon efficiencies for a variety of hydrocarbons, specifically in the case of the $C H$ Gerö band and of the $C_{2}$ Swan band, were measured in reference experiments in TEXTOR without surface contamination. The normalised photon-per-particle production efficiencies for $\eta(C I I) \simeq 0.5, \eta(C I) \simeq 0.2$ and $\eta\left(H_{\gamma}\right) \simeq 0.2$ as well as the observation of $\mathrm{CH}^{+}$during methane injection in TEXTOR indicate that at high electron temperatures the break-up chain, though a multi-step process, goes substantially via molecular ions. The TEXTOR benchmark experiment verified the current database for the methane break-up, used in different erosion/deposition models. An independent verification of the database was done at DIII-D [16]. 
A comparison of $\mathrm{D} / \mathrm{XB}$ values for methane, obtained in different machines as a function of $T_{e}$, was presented and compared to calculations with HYDKIN. The re-erosion of higher hydrocarbons built up during injection of methane was observed and its influence on the effective inverse photon efficiencies for methane discussed.

At JET, the part of the erosion yield associated with the production of $C_{2} H_{y}$ was measured in the outer divertor in L-mode: it was as low as $0.6 \%$. Improvements in the data analysis for chemical erosion yields were brought forth by TEXTOR. Higher hydrocarbons play a minor role. Physical and chemical erosion are balanced and each process lay about 3.0\% at the temperature maximum for chemical - hence total - erosion.

First results on hydrocarbon injections into the detached outer divertor of DIII-D and ASDEX Upgrade were shown, indicating a drastic reduction of the hydrocarbon flux by more than one order of magnitude during detachment, although higher effective inverse photon efficiencies were measured than predicted by HYDKIN. This regime has been identified as favourable for ITER and further experiments on different machines are foreseen to exploit its advantage of low chemical erosion.

This work was done in the frame of the European Task Force on Plasma-Wall Interaction.

\section{References}

[1] R. Pitts et al., Plasma Phys. Control. Fusion 47 (2005) B303

[2] V. Philipps et al., Plasma Phys. Control. Fusion 45 (2003) A17

[3] A. Kirschner et al., Nucl. Fusion 40 (2000) 989

[4] J. Roth et al., Nucl. Fusion 44 (2004) L21

[5] A. Pospieszczyk et al., J. Nucl. Mater. 145-147 (1987) 547

[6] http://adas.phys.strath.ac.uk

[7] S. Brezinsek et al., Plasma Phys. Control. Fusion 47 (2005) 615

[8] R. Janev and D. Reiter, Phys. Plasmas 9 (2002) 4071 
[9] A. Pospieszczyk et al., UCLA, Report PPG-125 (1989)

[10] S. Brezinsek et al., Physica Scripta T111 (2004) 42

[11] U. Fantz et al., J. Nucl. Mater. 337-339 (2005) 1087

[12] T. Nakano et al., Nucl. Fusion 42 (2002) 689

[13] R.C. Isler et al., Phys. Plasmas 8 (2001) 4470

[14] M.F. Stamp et al., Physica Scripta T91 (2001) 13

[15] P.T. Greenland and S. Brezinsek, FZJ - ISSN 0944-2952, Report Juel-4156 (2004)

[16] P. Stangeby et al., this conference

[17] M. Groth et al., this conference

[18] R. Pugno et al., J. Nucl. Mater. 337-339 (2005) 985

[19] A. McLean et al., this conference

[20] Y. Xu et al., this conference

[21] O. Schmitz et al., this conference

[22] http://www.eirene.de/eigen

[23] A. Huber et al., Physica Scripta T111 (2004) 101

[24] R. Pugno et al., 30th EPS Contr. Fus. and Plasma Phys., St. Petersburg (2003)

[25] S. Brezinsek et al., 32th EPS Plasma Phys., Tarragona (2005)

[26] P. Coad et al., this conference

[27] A. Pospieszczyk et al., J. Nucl. Mater. 241-243 (1997) 821

[28] D.G. Whyte et al., Nucl. Fusion 41 (2001) 1243 


\section{Tables}

Table 1

\begin{tabular}{|c||c|c|c|c|}
\hline \multirow{2}{*}{ species } & \multicolumn{2}{|c|}{$C H(C D)$ Gerö band } & \multicolumn{2}{c|}{$C_{2}$ Swan band } \\
\cline { 2 - 5 } & $\frac{D}{X B}$ EXP & $\frac{D}{X B}$ JR & $\frac{D}{X B}$ EXP & $\frac{D}{X B}$ JR \\
\hline \hline$C_{4} H_{4}$ & 27 & 37 & 1030 & - \\
\hline$C_{2} H_{4}$ & 35 & 53 & 40 & 25 \\
\hline$C_{2} H_{6}$ & 30 & 46 & 59 & 28 \\
\hline$C_{3} H_{8}$ & 19 & 28 & 41 & 20 \\
\hline \hline$C_{4} D_{4}$ & 36 & $(46)$ & 930 & - \\
\hline$C_{2} D_{4}$ & 31 & $(65)$ & 48 & $(33)$ \\
\hline$C_{2} D_{6}$ & 27 & $(57)$ & 65 & $(37)$ \\
\hline
\end{tabular}


Table 2

\begin{tabular}{|c|c|c|c|c|c|}
\hline experiment & species & $\operatorname{span}[\mathrm{nm}]$ & {$\left[\frac{D}{X B}\right]_{\mathrm{A}}{ }^{2} \Delta \rightarrow \mathrm{X}^{2} \Pi$} & $f\left(T_{\text {rot }}, \lambda_{\text {span }}\right)$ & {$\left[\frac{D}{X B}\right]_{\mathrm{A}^{2} \Delta \rightarrow \mathrm{X}^{2} \Pi}^{\text {full range }}$} \\
\hline ASDEX Upgrade [11] & $\mathrm{CH}$ & $430.0-431.5$ & 154 & 2.8 & 55 \\
\hline ASDEX Upgrade [23] & $\mathrm{CH}$ & $430.0-431.5$ & $11-56$ & 2.8 & $4-20$ \\
\hline DIII-D [17] & $\mathrm{CH}$ & $427.0-431.5$ & 71 & 1.8 & 39 \\
\hline $\mathrm{JET}[10]$ & $\mathrm{CD}$ & 429.4-430.9 & $154-168$ & 2.8 & $55-60$ \\
\hline JET [22] & $\mathrm{CD}$ & $427.8-431.5$ & 100 & 1.9 & 53 \\
\hline JET [14] & $\mathrm{CD}$ & $427.8-431.5$ & 80 & 1.9 & 42 \\
\hline TEXTOR [23] & $\mathrm{CH}$ & $430.0-431.5$ & $100-240$ & 2.8 & $32-110$ \\
\hline TEXTOR [here] & $\mathrm{CD}$ & $429.5-431.0$ & 101 & 2.8 & 36 \\
\hline TEXTOR [here] & $\mathrm{CH}$ & $430.0-431.5$ & 76 & 2.8 & 27 \\
\hline
\end{tabular}




\section{Table captions}

Table 1: Break-up of different types of $C_{x} H_{y}$ in $D$ plasmas $\left(T_{e}=35 \mathrm{eV}\right)$ and $C_{x} D_{y}$ in $H$ plasmas $\left(T_{e}=45 \mathrm{eV}\right)$. Effective $\mathrm{D} / \mathrm{XB}$ values are related to the full emission range. Values in brackets are calculated under the assumption of ordinary hydrocarbon injection. Table 2: D/XB value normalisation: $f\left(T_{\text {rot }}, \lambda_{\text {span }}\right)$ values for the Gerö band in different experiments with $\mathrm{CD}_{4}$ or $\mathrm{CH}_{4}$ injection (at different $T_{e}$ values).

\section{Figure captions}

Figure 1: a) Measured and modelled spectra of the A-X band of $C D^{+}$and $C D$. b) No difference in the ro-vibrational population of the $C_{2}$ Swan band for similar plasmas.

Figure 2: $C D_{4}$ injection in TEXTOR: a) 2D pattern of $C D$ light, b) Penetration depth for $C_{2}, C I I, C D$ and $D_{\gamma}$, and c) ERO modelling of the $C D$ light pattern.

Figure 3: Normalised $\left[\frac{D}{X B}\right]_{\mathrm{A}}{ }^{2 H_{4} \rightarrow C H} \mathrm{X}^{2} \Pi$ observed in different fusion devices and calculated values from HYDKIN as function of $T_{e}$.

Figure 4: Observation of $\phi_{C_{2}}$ during $C_{4}$ injection in ASDEX Upgrade.

Figure 5: In-situ determination of $Y_{C}^{\text {chem }}$ attributed to $C_{2} H_{y}$ in JET.

Figure 6: Determination of $Y_{C}^{\text {chem }}$ and $Y_{C}^{\text {phys }}$ by simultaneous observation of $C H, C I I$ and $D_{\gamma}$ in TEXTOR.

Figure 7: Gerö band in the outer divertor of ASDEX Upgrade under attached and detached (w/wo methane injection) plasma conditions. 


\section{Figures}
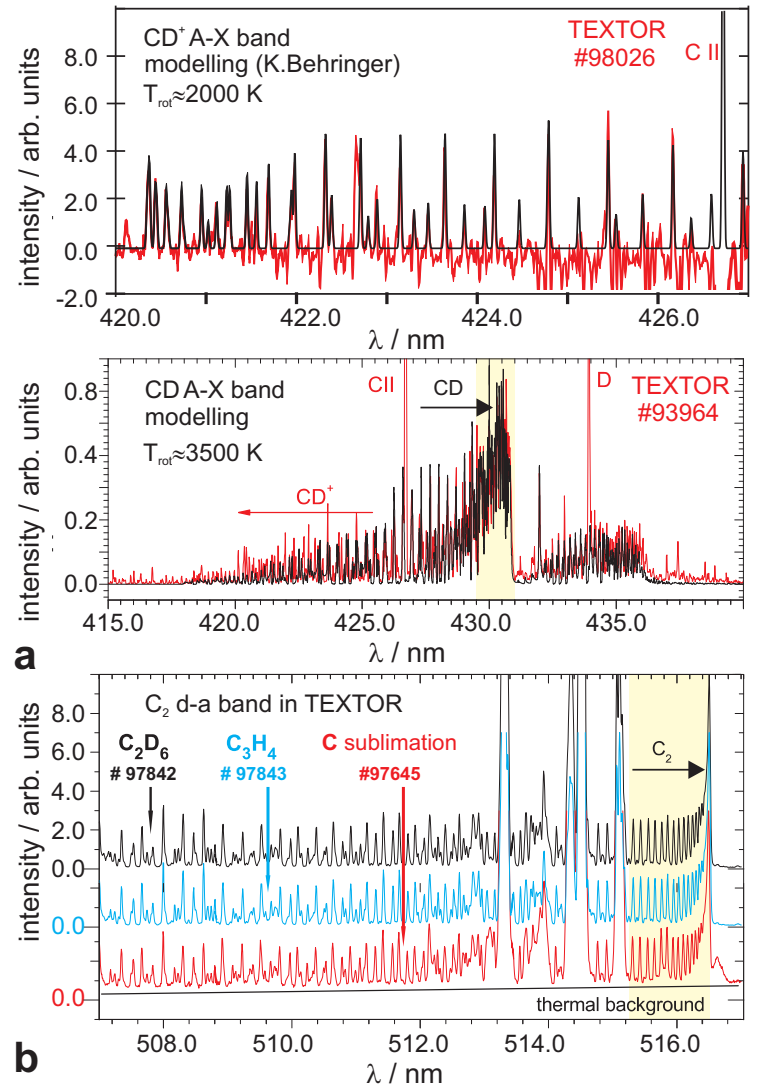

Figure 1: 

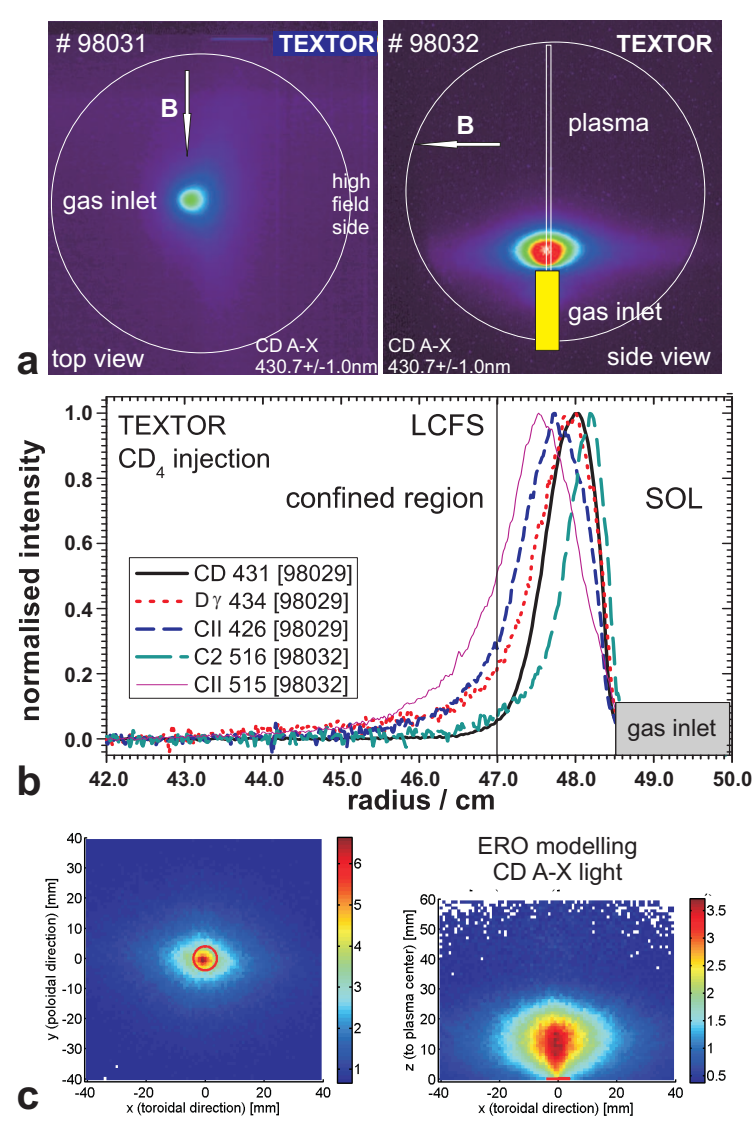

Figure 2: 


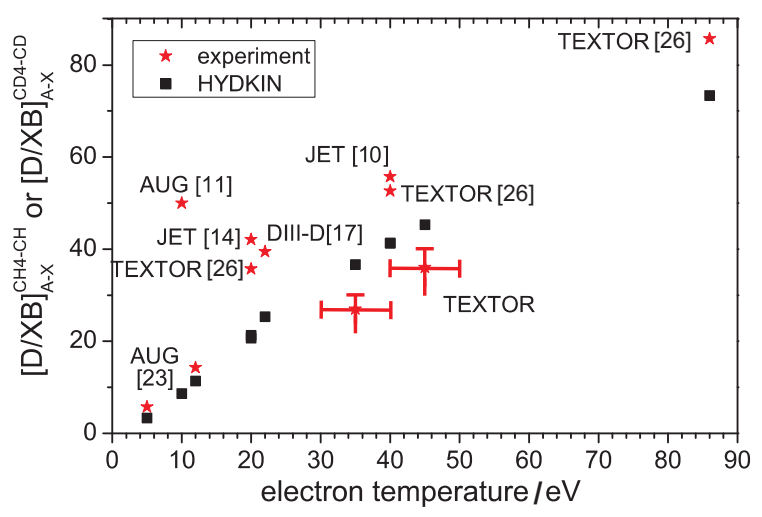

Figure 3: 


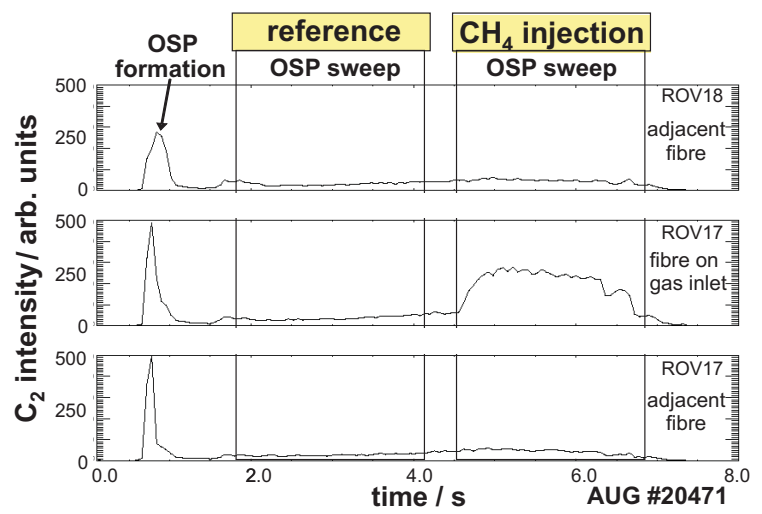

Figure 4: 


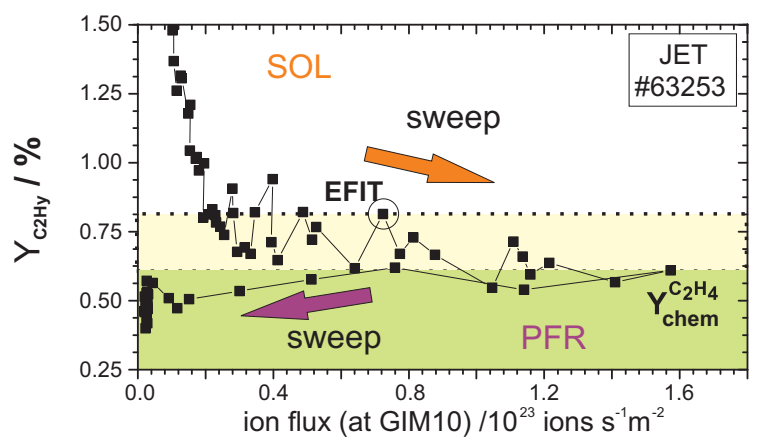

Figure 5: 

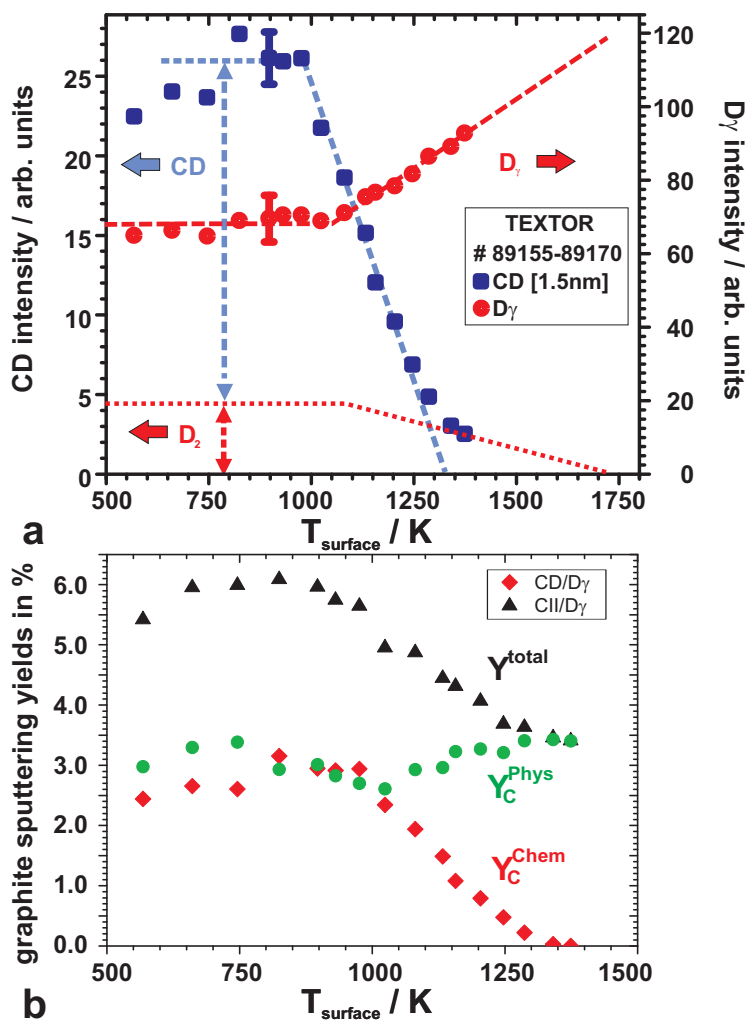

Figure 6: 


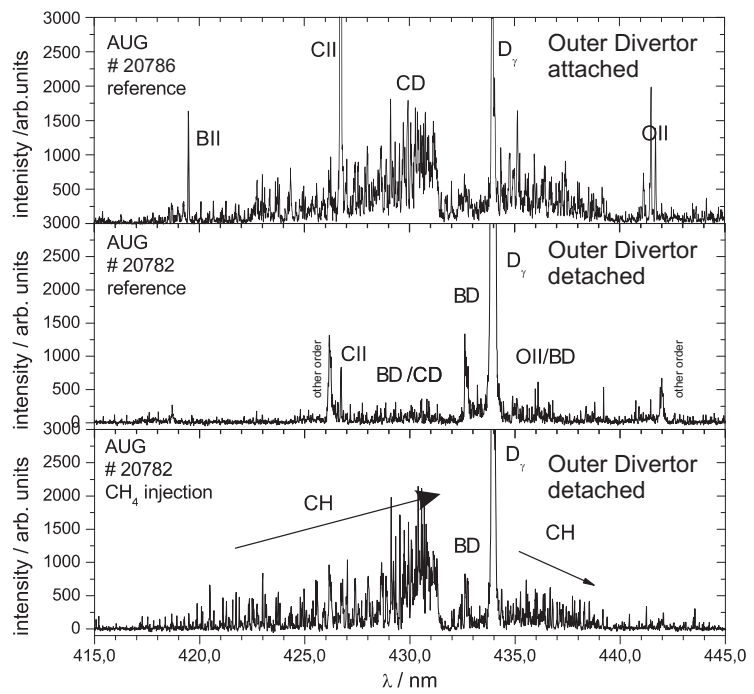

Figure 7: 